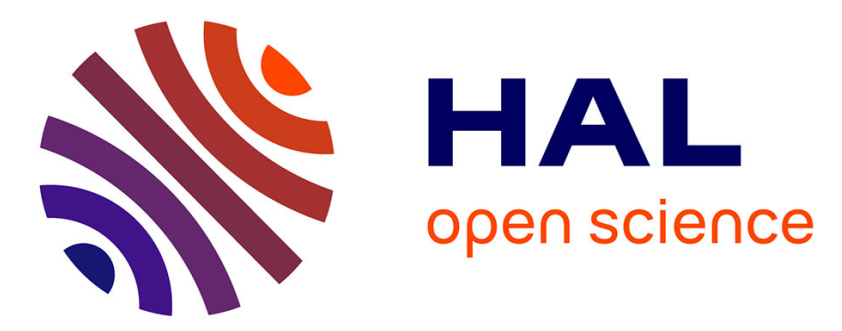

\title{
Dedicating Finite Volume Method to Electromagnetic Plasma Modeling: Circuit Breaker Application
}

\author{
Loïc Rondot, Vincent Mazauric, Yves Delannoy, Gérard Meunier
}

\section{To cite this version:}

Loïc Rondot, Vincent Mazauric, Yves Delannoy, Gérard Meunier. Dedicating Finite Volume Method to Electromagnetic Plasma Modeling: Circuit Breaker Application. IEEE Transactions on Magnetics, 2009, 45 (3), pp 1262-1265. hal-00404047

\section{HAL Id: hal-00404047 \\ https://hal.science/hal-00404047}

Submitted on 15 Jul 2009

HAL is a multi-disciplinary open access archive for the deposit and dissemination of scientific research documents, whether they are published or not. The documents may come from teaching and research institutions in France or abroad, or from public or private research centers.
L'archive ouverte pluridisciplinaire $\mathbf{H A L}$, est destinée au dépôt et à la diffusion de documents scientifiques de niveau recherche, publiés ou non, émanant des établissements d'enseignement et de recherche français ou étrangers, des laboratoires publics ou privés. 


\title{
Dedicating Finite Volume Method to Electromagnetic Plasma Modeling: Circuit Breaker Application
}

\author{
Loïc Rondot ${ }^{1,2,3}$, Vincent Mazauric ${ }^{1}$, Yves Delannoy ${ }^{2}$, and Gerard Meunier ${ }^{3}$ \\ ${ }^{1}$ Schneider Electric Science and Technology, 38050 Grenoble cedex 9, France \\ ${ }^{2}$ SIMAP, Science \& Engineering for MAterials and Proceeding 38402 Grenoble, France \\ ${ }^{3}$ G2Elab-Grenoble Electrical Engineering LAB, 38402 Grenoble, France
}

\begin{abstract}
For several applications including strong hydrodynamics constraints (like in a circuit breaker), Finite Element solvers are not suitable for fluid dynamical modeling. For these applications, the coupling between the Computational Fluid Dynamics and the electromagnetism modeling is not easy. In this context, a magnetodynamic formulation is developed using the Finite Volume Method to address specific issues in multiphysics involved in circuit breaker modeling. The principles of Finite Volume Method, their using for the electromagnetism modeling, and multiphysics coupling method are described. Problems are expressed within current based $T-T_{0}-\phi$ formulation which differs drastically from other developments given in the context of Finite Volume Method. Finally a circuit breaker simulation is carried out within a magnetostatic formulation.
\end{abstract}

Index Terms-Circuit breaker, finite volume method, magneto-hydrodynamic, plasma.

\section{INTRODUCTION}

$\mathbf{V}$ ARIOUS multiphysic modeling, and more particularly the current breaking modeling, requires fluid dynamics and electromagnetic models [1]. Whereas the conventional finite volume method (FVM) usually dedicated to computational fluid dynamics enforces the local conservation of mass, momentum and energy [2], low-frequency electromagnetic software minimizes global energy functionals within the finite element method (FEM) [3]. Hence, magnetohydrodynamics problems are currently resolved by using either:

- a FE-CFD code, which is not suitable for circuit breaker applications because the method is unable to model the shock waves during the breaking process, or

- a hybrid method combining a FVM and a FEM, thereby sacrificing the high level of integration and the accuracy achieved with a single mesh [4].

Thus, the search for a common, effective and integrated model and important hydrodynamic constraints call for a single numerical method for the two phenomena. In this work, an electromagnetic model based on the FVM is adopted.

\section{Electromagnetism Within the Finite VOLUME METHOD}

\section{A. Finite Volume Method}

The Finite Volume Method is based on the local conservation (Fig. 1). Expressing the balance in any elementary volume

Manuscript received October 07, 2008. Current version published February 19, 2009. Corresponding author: L. Rondot (e-mail: loic.rondot@yahoo.fr).

Color versions of one or more of the figures in this paper are available online at http://ieeexplore.ieee.org.

Digital Object Identifier 10.1109/TMAG.2009.2012587

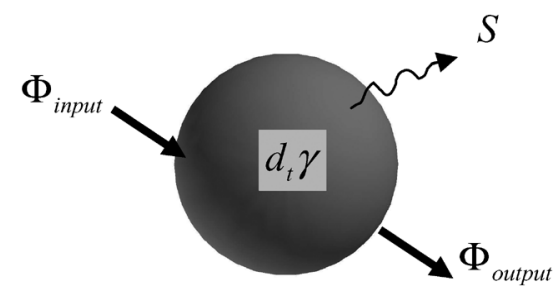

Fig. 1. Local balance of the flow $\Phi: d_{t} \gamma$ is the local variation and $S$ denotes the source term.

between the dissipative term (RHS) and the variation over time and the flux exchanged at its boundary (LHS), it reads:

$$
\underbrace{\partial_{t} \gamma}_{\text {unsteady term }}+\underbrace{\operatorname{div}(-k \operatorname{grad} \gamma+\mathbf{v} \gamma)}_{\text {diffusive and convective flux term }}=\underbrace{S}_{\text {source term }}
$$

where $\gamma$ denotes the transported physical quantity, $k$ is the diffusion coefficient of the transported quantity and $\mathbf{v}$ its velocity.

In FEM, the unknowns are associated to the nodes or edges of the mesh and the integral approximation is made by an approximation of the solution. In finite volume method, the unknowns are on the cell centroid and the integral approximation is made by an operator approximation as shown in (2). The diffusive flux $D_{f}$, across a face $f$ of a scalar $V$ is given by:

$$
\begin{aligned}
D_{f} & =\iiint \operatorname{div}(-k \nabla V) d \Omega \\
& =\underbrace{-k \frac{V_{1}-V_{0}}{d s} \frac{\mathbf{A} \cdot \mathbf{A}}{\mathbf{A} \cdot \mathbf{e s}}}_{\text {Primary flux(implicit term) }}+\underbrace{k \nabla V \cdot\left(\mathbf{A}-\mathbf{e s} \frac{\mathbf{A} \cdot \mathbf{A}}{\mathbf{A} \cdot \mathbf{e s}}\right)}_{\text {Secondary flux(explicit term) }}
\end{aligned}
$$

where $k$ is the diffusion coefficient at the face, $V_{0}$ and $V_{1}$ the scalar value in the cell $c_{0}$ and $c_{1}, \mathbf{A}$ is the area normal vector of face directed from cell $\mathrm{c}_{0}$ to $\mathrm{c}_{1}, d s$ is the distance between the cell centroids, es is the unit normal vector in this direction (Fig. 2).

Whereas the first term of $D_{f}$ on the right hand side represents the primary gradient directed along the vector es and the 


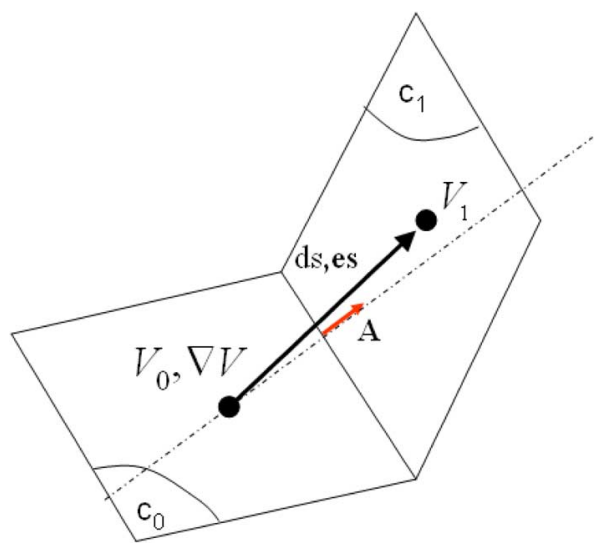

Fig. 2. Diffusive flux approximation for non-Cartesian meshes.

second term represents the cross diffusion term directed along the vector $\mathbf{e s}^{\perp}$. While the former is an implicit term, the latter is explicit and allows to correct fluxes for non-Cartesian meshes, latter the former implicit.

In the following, the previous description is adopted successfully to solve electromagnetic elementary problem within the so-called $\mathbf{T}-\mathbf{T}_{\mathbf{0}}-\phi$ formulation. This formulation is known to use a decreasing number of degrees of freedom compared to the magnetic potential vector formulation [5].

\section{B. Electromagnetism Modeling}

1) Static Current Flow (3-D): Static current flow modeling is straightforward. The current flux divergence free follows a classical thermal diffusion equation:

$$
\operatorname{div}(-\sigma \nabla V)=0
$$

where $\sigma$ is the electrical conductivity and $V$ is is the electrical potential.

When there is a strong variation of electrical conductivity, results are improved with a current density vector reconstruction by a least square method based on the current fluxes. This method is more relevant than a classical using of the electrical potential gradient crossed by the electrical conductivity.

2) Vacuum Magnetostatics (3-D): In order to determine the field $\mathbf{T}_{\mathbf{0}}$ related to the current sources, we focus on the curl of current density. By using the gauge $\operatorname{div} \mathbf{T}_{\mathbf{0}}=0$ and Faraday's law for static regime, $\mathbf{T}_{\mathbf{0}}$ checks:

$$
-\Delta \mathrm{T}_{0}=\text { curlJ }
$$

To circumvent the difficulty of calculating curl $\mathbf{J}$ in a finite volume framework, it is convenient to transform (4) along a divergent form. As a result, the vectorial problem (4) is transformed into three scalar problems coupled only through the boundary conditions of far field.

This formulation is applied to the case of a 3-D cylindrical conductor supplied by a current and validated with the CFD Fluent code.

The comparison with the analytic solution shows a reasonable error which is located mainly in the zone of weak field (Fig. 3). Such an error may be reduced thanks to a domain extension.

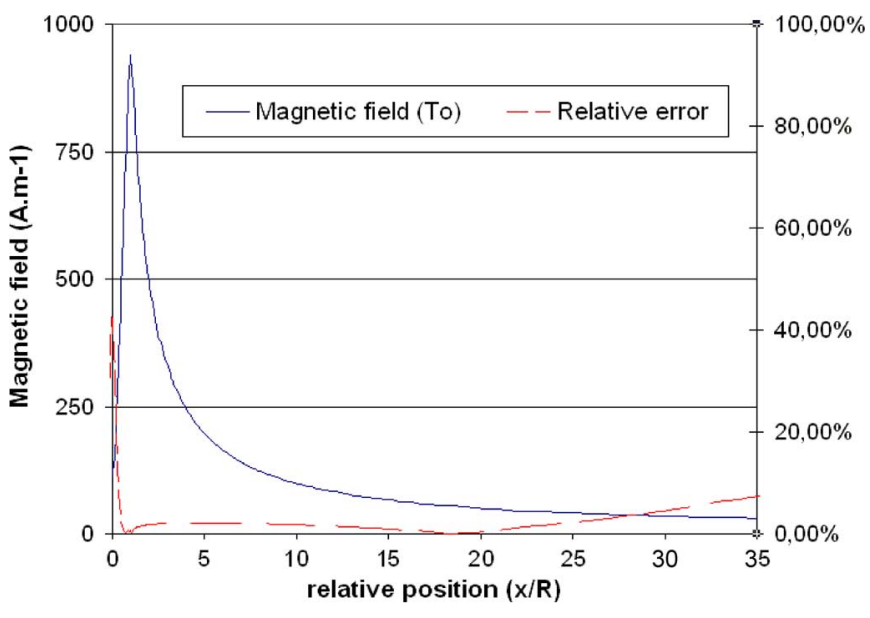

Fig. 3. Orthoradial field created by a cylindrical conductor supplied by an even current (solid line) and error introduced by a FV calculation in comparison to the analytical solution (dashed).

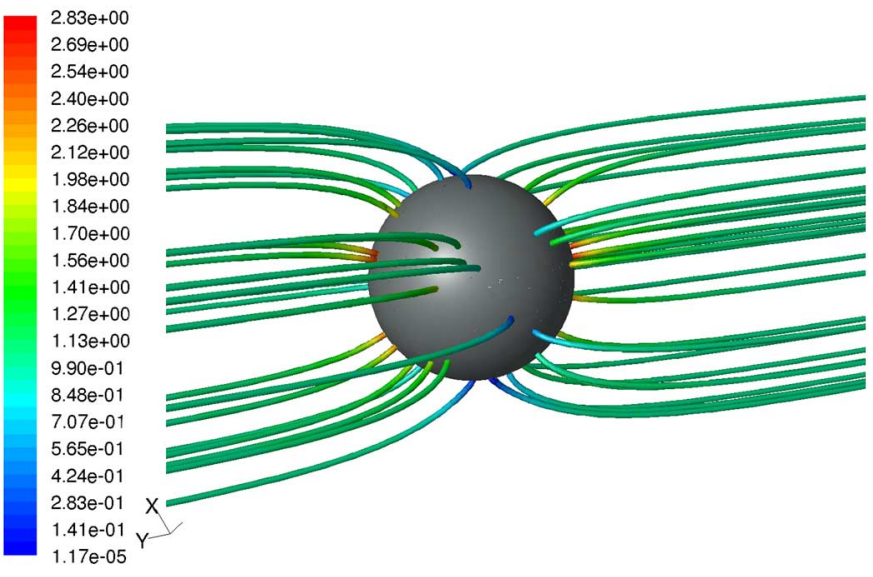

Fig. 4. H-line refraction around the magnetic sphere $\left(\mu_{r}=1000\right)$.

3) Magnetostatics (3-D): For 3-D magnetostatics using a $\mathbf{T}_{\mathbf{0}}-\phi$ formulation, the magnetic field reads $\mathbf{H}=\mathbf{T}_{\mathbf{0}}-\boldsymbol{\nabla} \phi$, and the magnetic flux density divergence-free is expressed as a diffusion equation with a source term resulting from the field obtained in the vacuum case:

$$
\underbrace{\operatorname{div}(-\mu \nabla \phi)}_{\text {diffusive term }}=\underbrace{\operatorname{div}\left(-\mu \cdot \mathbf{T}_{0}\right)}_{\text {source term }}
$$

This formulation is used in the case of a magnetic 3-D sphere plunged in a uniform field $\mathbf{T}_{\mathbf{0}}$. The concentration of the magnetic flux in the vicinity of the sphere (Fig. 4) is observed qualitatively. Moreover, for $\mu_{r}$ lower than 10000 , the assessment of the magnetic energy within the sphere deviates from analytic solution [6] less than 3\% (Fig. 5).

For higher values of $\mu_{r}$, the cancellation error related to the reduced scalar potential formulation appears [7]. As the magnetic permeability of iron pieces is about 1000 in circuit breakers, the method is suitable for this kind of modeling.

4) Magnetodynamics (3-D): When transient effects are taken into account, the previous definition of the magnetic field is enhanced in the conductors thanks to an extra term $\mathbf{T}$ related to 


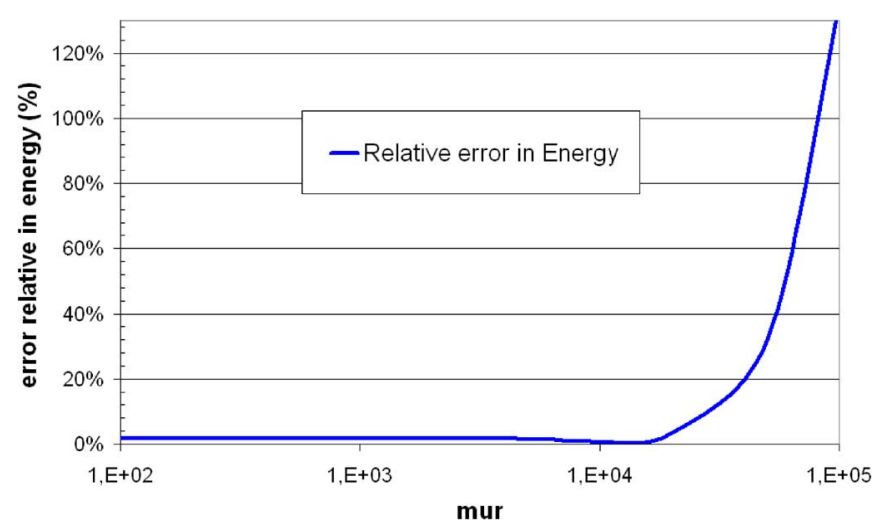

Fig. 5. Relative error in energy introduced by the FVM (right).

the eddy currents and follow: $\mathbf{H}=\mathbf{T}+\mathbf{T}_{\mathbf{0}}-\operatorname{grad} \phi$. For homogeneous conductors, Faraday's equation, Ohm's law and the gauge $\operatorname{div} \mathbf{T}=0$ yield the relation (6) providing the evolution of $T$ :

$$
-\Delta \mathbf{T}+\sigma \partial_{t}\left(\mu\left(\mathbf{T}+\mathbf{T}_{0}-\nabla \phi\right)\right)=0
$$

This non-stationary diffusion equation needs particular boundary conditions:

- Current conservation is enforced thanks to

$$
\mathbf{T} \times \mathbf{n}=\mathbf{0}
$$

- To ensure the equivalence between the (6) and the Faraday's law, the gauge should be satisfied [8]. Combination of the gauge and (7) give

$$
\partial_{n}(\mathbf{T} \cdot \mathbf{n})=0 .
$$

The $\mathbf{T}-\mathbf{T}_{\mathbf{0}}-\phi$ formulation along with boundary conditions is applied to a circuit including two parallel ferromagnetic cylinders $\left(\mu_{r}=500\right)$ supplied by a transient current (Fig. 6.)

Comparison with finite element method performed with Flux ${ }^{\circledR}$ CAO Software is lower than 5\% in energy. However, the convergence of finite volume method is time-consuming due to the segregated resolution on each $\mathbf{T}$ component.

\section{Circuit BREAKer Modeling}

In the breaking process, the transient effects can be neglected in a first approach [9]. Therefore it is possible to model the process within the previous studies FV electromagnetic developments.

\section{A. The Framework of the Resolution Procedure}

In Fig. 7, the framework of the resolution procedure is represented for one iteration.

The previous formulation was implemented within the plasma physics-dedicated Schneider Electric software. This code already included:

- a real gas model;

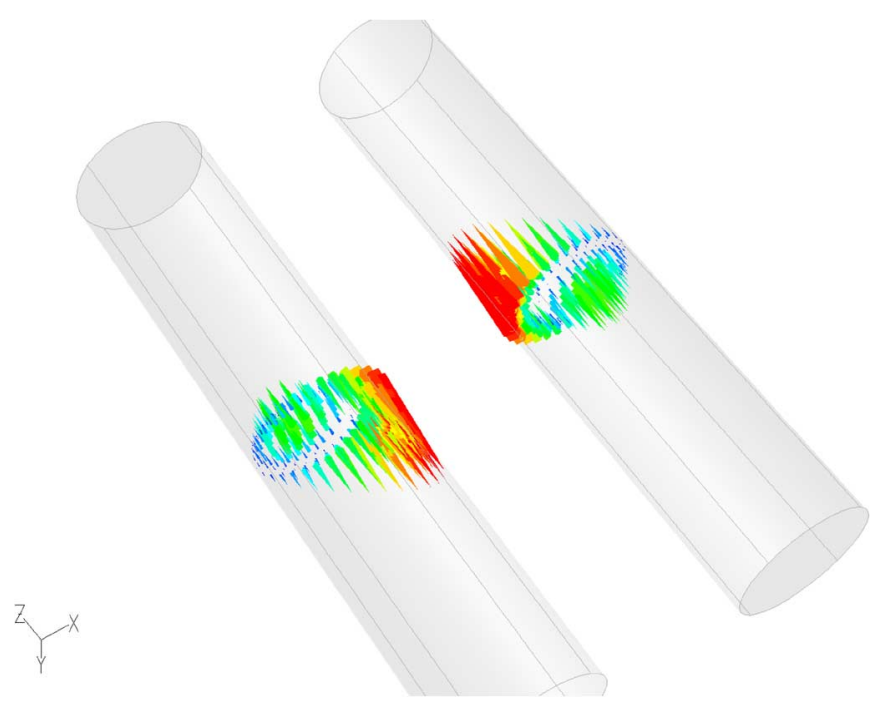

Fig. 6. Eddy current in two ferromagnetic cylinders $\left(\mu_{r}=500\right)$ supplied by a transient current.

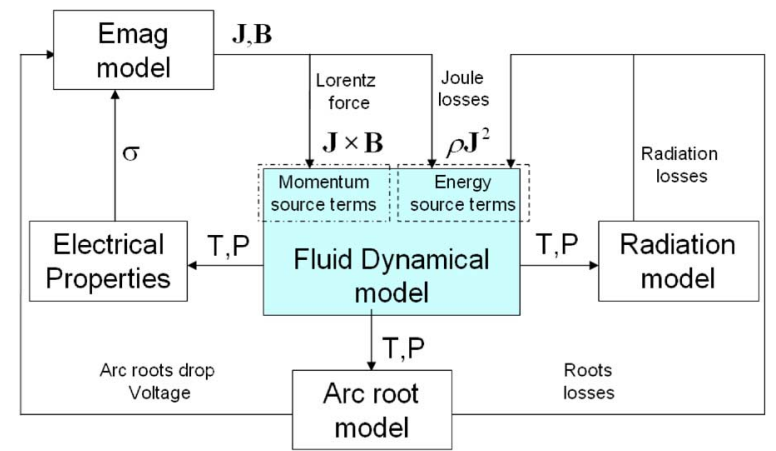

Fig. 7. Chart of the resolution procedure for the breaking process modeling (T: temperature, $\mathrm{P}$ : pressure, $\sigma$ : electrical conductivity).

- a radiation model;

- a table of electrical properties;

- an arc root model.

All the models (real gas, radiation, root model and electromagnetism) are driven by the fluid dynamical based core [10]. These models are the inputs of source terms in energy and momentum. In the case of electromagnetism, the Lorentz forces and the Joule losses are introduced in fluid dynamical solver (Fig. 7).

This resolution with the CFD Fluent code is achieved thanks to its explicit solver, which uses a Gauss-Seidel method with a multi-grid resolution.

\section{B. Circuit Breaker Modeling}

In Fig. 8, an experimental mock-up is modeled. The mock-up is composed of two runners, a mobile contact and a far pressure outlet. The mesh has 400000 cells and the unknown solving are $\left(\rho, \mathbf{v}, \mathrm{H}, V, \mathbf{T}_{\mathbf{0}}, \phi\right)$, respectively density, velocity, Gibbs energy, electrical potential, field in the vacuum and magnetic scalar potential. The computational time for a breaking process modeling is about 3 weeks. 


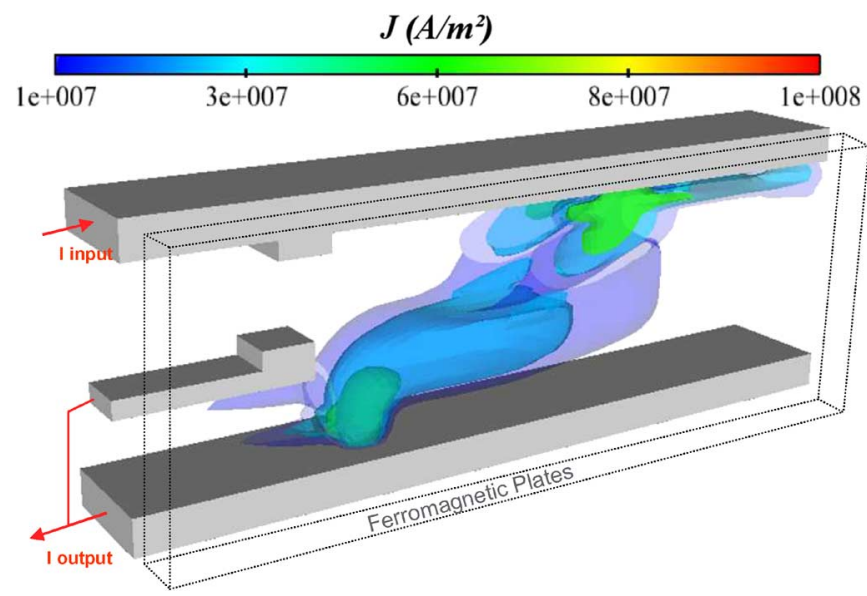

Fig. 8. Three iso-values of current density in a circuit breaker (left).

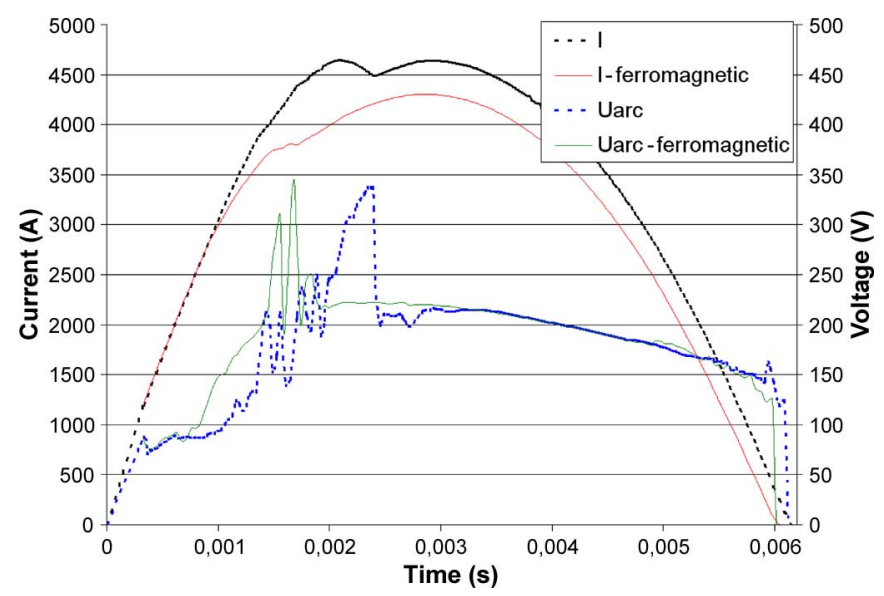

Fig. 9. While the two upper curves represent the current-limited waves, the two lower curves provide the arc voltage drop. As a result, the ferromagnetic plates enhance the arc motion.

In Fig. 8, three iso-values of current density in a circuit breaker when the arc is commuting are shown. The field is found greater around the arc and in the vicinity of the runners.

In order to model the influence of ferromagnetic materials, two ferromagnetic plates are introduced along the mock-up. The breaking process is compared with the previous simulation.

As a result, the arc motion is enhanced, so that the ferromagnetic plates act as flux concentrators.
By inspection of Fig. 9, the arc grows quickly and so does the arc voltage with ferromagnetic parts. The current undergoes an extra limitation and the zero is obtained faster so that the breaking process.

\section{CONCLUSION}

Within sight of the results obtained with magnetostatic cases, the finite volume method seems suitable to model efficiently electromagnetism. This method does not have any ambition to compete with finite element method but simply to allow a stronger coupling between the fluid dynamical and electromagnetism in the specific case of breaking operation. These electromagnetic developments allow already to model the breaking process with ferromagnetic materials. The comparison with the experimental data is not easy but is under progress. The nonlinear ferromagnetic material modeling is already efficient but the CPU time is too important to be used in the total breaking process modeling. Subsequent improvements and productivity in the design of breaking products (MV and LV circuit breaker) are expected.

\section{REFERENCES}

[1] C. A. Borghi and A. Cristofolini, "A hybrid implicit numerical method for the analysis of the magneto-plasmadynamics in a gas discharge," IEEE Trans. Magn., vol. 40, 2004.

[2] S. V. Patankar, Numerical Heat Transfer and Fluid Flow. New York: Hemisphere Publishing Corp., 1983.

[3] V. Mazauric, "From thermostatistics to Maxwell's equations: A variational approach of electromagnetism," IEEE Trans. Magn., vol. 40, pp. 945-948, 2004.

[4] I. J. Lyttle, "Use of MPCCI to perform multidisciplinary analyses for electrical distribution equipment," American Inst. Aeronautics and Astronautics, paper 0122,2005 .

[5] M. Springstubbe and M. Lindmayer, "Three-dimensional-simulation of arc motion between arc runners including the influence of ferromagnetic material," IEEE Trans. Compon. Packag. Technol., vol. 25, p. 409, 2002.

[6] J. A. Osborn, "Demagnetizing factors of the general ellipsoid," Phys. Rev., vol. 67, no. 11 \& 12, 1945.

[7] K. Sivasubramaniam, S. Salon, and M. V. K. Chari, "Numerical errors in the scalar potential formulations used in low-frequency field problems," IEEE Trans. Magn., vol. 36, pp. 3096-3098, 2000.

[8] O. Biro, K. Preis, W. Renhart, G. Vrisk, and F. R. Richter, "Computation of 3-D current driven skin effect problems using a current vector potential," IEEE Trans. Magn., vol. 24, pp. 1325-1328, 1993.

[9] O. Chadebec, G. Meunier, V. G. Mazauric, Y. Le Floch, and Y. Labie, "Eddy-current effects in circuit breakers during arc displacement phase," IEEE Trans. Magn., vol. 40, pp. 1358-1361, 2004.

[10] F. Karetta and M. Lindmayer, "Simulation of the gasdynamic and electromagnetic processes in low voltage switching arcs," IEEE Trans. Compon., Packag., Manufact. Technol.-Part A, vol. 21, pp. 96-103, 1998. 